\title{
Speckle Reduction for LIDAR Using Optical Phase Conjugation
}

M. W. Bowers, C. Kecy, L. Little, J. Cooke, J. Benterou, R. Boyd, and T. Birks

February 26, 2001

U.S. Department of Energy

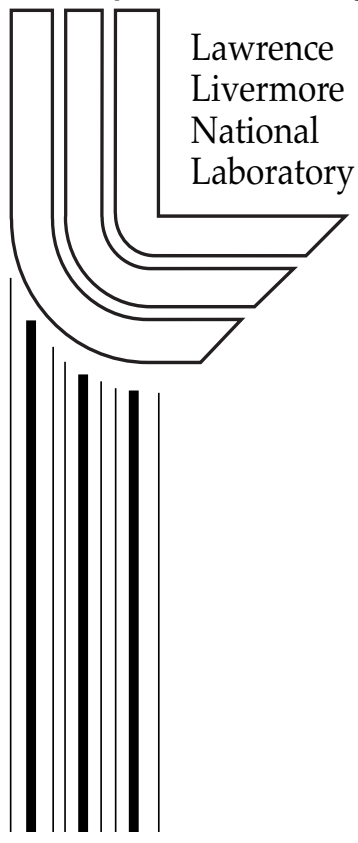




\section{DISCLAIMER}

This document was prepared as an account of work sponsored by an agency of the United States Government. Neither the United States Government nor the University of California nor any of their employees, makes any warranty, express or implied, or assumes any legal liability or responsibility for the accuracy, completeness, or usefulness of any information, apparatus, product, or process disclosed, or represents that its use would not infringe privately owned rights. Reference herein to any specific commercial product, process, or service by trade name, trademark, manufacturer, or otherwise, does not necessarily constitute or imply its endorsement, recommendation, or favoring by the United States Government or the University of California. The views and opinions of authors expressed herein do not necessarily state or reflect those of the United States Government or the University of California, and shall not be used for advertising or product endorsement purposes.

This work was performed under the auspices of the U. S. Department of Energy by the University of California, Lawrence Livermore National Laboratory under Contract No. W-7405-Eng-48.

This report has been reproduced directly from the best available copy.

Available electronically at http://www.doc.gov/bridge

Available for a processing fee to U.S. Department of Energy

And its contractors in paper from

U.S. Department of Energy

Office of Scientific and Technical Information

P.O. Box 62

Oak Ridge, TN 37831-0062

Telephone: (865) 576-8401

Facsimile: (865) 576-5728

E-mail: reports@adonis.osti.gov

Available for the sale to the public from

U.S. Department of Commerce

National Technical Information Service

5285 Port Royal Road

Springfield, VA 22161

Telephone: (800) 553-6847

Facsimile: (703) 605-6900

E-mail: orders@ntis.fedworld.gov

Online ordering: http://www.ntis.gov/ordering.htm

OR

Lawrence Livermore National Laboratory

Technical Information Department's Digital Library

http:/ / www.llnl.gov/tid/Library.html 


\title{
SPECKLE REDUCTION FOR LIDAR USING OPTICAL PHASE CONJUGATION
}

\author{
Mark W. Bowers - PI \\ Chad Kecy, Liesl Little, Jeff Cooke, Jerry Benterou - LLNL co-investigators \\ Professor Robert Boyd - University of Rochester \\ Professors Tim Birks - University of Bath
}

\begin{abstract}
Remote detection of chemicals using LIDAR (Light Detection and Ranging) utilizing DIAL (Differential Absorption LIDAR) is now a standard detection technique for both military and civilian activities. We have developed a novel nonlinear optical phase conjugation system that can reduce the effects of speckle noise and atmospheric turbulence on DIAL remote detection systems. We have shown numerically and experimentally that it is possible to increase the signal-to-noise ( $\mathrm{S} / \mathrm{N}$ ) ratio for LIDAR systems under certain conditions using optical phase conjugation. This increase in $S / N$ can result in more accurate detection of chemical effluents while simultaneously reducing the time necessary to acquire this information.
\end{abstract}

\section{INTRODUCTION}

Remote detection systems can be used to detect and monitor chemical spills, the production of weapons of mass destruction (WMD), as well as environmental conditions around the world. It is imperative that these detection systems work reliably and accurately while being at a safe distance from the source. It would be advantageous if, in addition, the remote detection systems were compact and consumed little power so that they could be carried in smaller vehicles, planes, or even spacecraft. LIDAR (Light detection and ranging) is well suited for these applications. A LIDAR system is one in which a laser beam is transmitted out towards an area to be probed. The return scatter from the aerosol itself or the topographic background is detected and measured.

LIDAR systems can measure chemical effluents accurately by using a detection technique known as differential absorption LIDAR (DIAL). In the DIAL technique two or more laser pulses of different frequencies are transmitted sequentially towards a target area. The differences in return signal power for each of these frequencies is measured. Since it is assumed that the returned power would be identical for all frequencies in the absence of absorption, this differencing technique can determine the relative absorption at the transmitted frequencies.

In practical, field operational systems, the simplistic view given above is complicated by noise sources, such as speckle. Speckle, as shown in figure 1a, results from the light scattering off a diffuse surface. The light returning to any one point in the detector aperture will originate from multiple points on the diffuse surface. Each of these individual light rays will add up constructively or destructively depending upon its arrival 
phase. The random phases of each light ray will result in a random, but deterministic, light irradiance at each point in the detector aperture. If viewed with a camera, this pattern will appear as bright and dark spots, figure $1 \mathrm{~b}$. The exact number of bright and dark spots in the detector aperture is a random number, although the mean value and variance can be calculated for a given situation. Thus, the total irradiance measured by the detector is random, resulting in an unknown deviation from the expected signal return. The atmosphere will complicate even this picture by adding turbulence that can change the speckle return over time, usually over a few milliseconds time period.

(a)

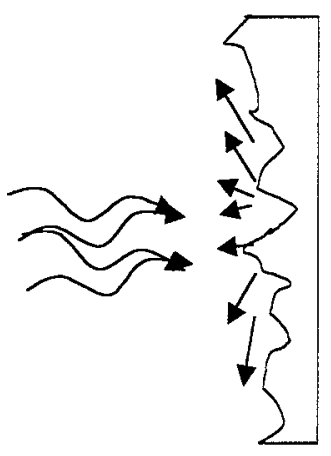

(b)

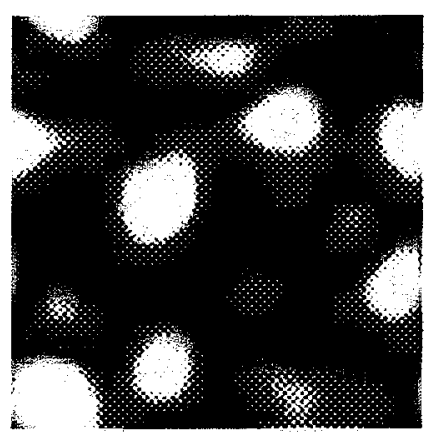

(c)

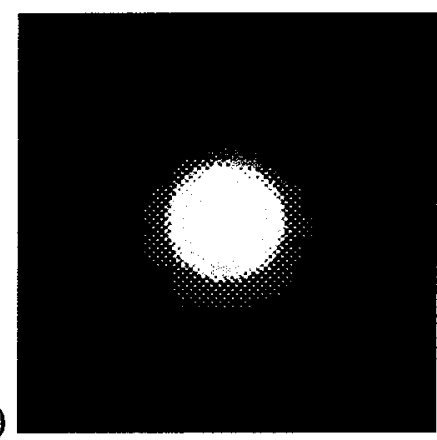

Figure 1. Random surfaces (a) result in optical interference on coherent LIDAR beams. A typical optical pattern with speckle noise (b) as viewed at the LIDAR receiver. An ideal LIDAR return signal as viewed at the focus of the receiver (c) would be uniform and symmetric.

The noise contributed to the DIAL signal by speckle and atmospheric turbulence can be reduced significantly, but at a cost. The mean and the variance of the signal are deterministic, and thus can be reduced by averaging. Averaging can improve the signalto-noise ratio $(\mathrm{S} / \mathrm{N})$ by an amount proportional to the square root of the number of pulses averaged. Since each pulse must wait at least long enough for the speckle field to change, the time between each pulse will be on the order of milliseconds. In order to get adequate results, averaging times of seconds or minutes may be necessary. The maximum averaging time is also limited by slow system drifts and will limit the accuracy of the resultant measurements to something well below that which is necessary to complete the desired mission.

Optical phase conjugation is a nonlinear optical method of removing the phase differences of the diffuse rays of light described above. By removing the phase differences, light can be made to sum constructively over the entire detector aperture, thus removing the presents of a speckle pattern and the associated noise. In order for optical phase conjugation to completely compensate for the speckle noise, all of the light over two-pi steradians must be collected and conjugated. This is not possible for LIDAR systems due to the large distances present between the LIDAR source and the topographic background. Previously it was generally accepted that capturing only a portion of the scattered light would, in most circumstances, completely null out the effects of the process. This belief was based on the premise that microscopic scattering dominates the phase-front of the optical beam propagation. Unpublished numerical 
studies have shown that the macroscopic scattering properties of a surface can be just as important in the wavefront evolution. Microscopic and macroscopic here refer to feature sizes smaller than or larger than the diffraction limited optical spot sizes respectively.

\section{THEORETICAL DESCRIPTION}

There are several types of optical phase conjugation that can accomplish nearly ideal phase front reversal. DIAL systems, however, require optical pulses of several hundred microjoules or more over many tens of nanoseconds. The high spatial frequency content of the DIAL return signal beam must also be considered when selecting the method of phase conjugation. Stimulated Brillouin scattering (SBS) is well suited for these conditions and was selected as the method of choice for this reason.

SBS results from the interaction of light with sound waves in a transparent medium. The process can be self-generating from a single input beam or can be enhanced through a secondary pump beam known as Brillouin-enhanced four-wave mixing (BEFWM). Both of the processes can result in efficient reflectivities. Single beam SBS reflectivities can approach $100 \%$ while BEFWM can have reflectivities exceeding $60 \mathrm{~dB}$ through pump energy exchange. Subsequent SBS gain stages can increase the energy in the conjugate beam by nearly $80 \mathrm{~dB}$ per stage [1]. These high gains are required to compensate for the losses experienced through the Lambertian-like scattering from the target and subsequent propagation through long paths in the atmosphere.

In order for SBS to compensate for the speckle the optical beam must pass twice through the distorting regions. This situation is shown in figure 2 for a LIDAR system. A low power probe beam, in this case it is a continuous wave (CW) laser, is transmitted out a LIDAR telescope. This beam is then incident upon a target area several kilometers distant. The probe beam is then diffusely reflected into $2 \pi$ steradians. A second telescope near the transmitting telescope captures some of this beam. The probe beam has now been attenuated $80-100 \mathrm{~dB}$ from its transmitted power due to the diffuse reflection and distance of propagation. The beam is then directed through an SBS amplifier and into a BEFWM oscillator. A pulsed laser is directed into the same BEFWM oscillator. The Brillouin interaction of the two beams in this oscillator results in the $\mathrm{CW}$ signal beam being phase conjugated during the time interval of the pump laser pulse. In fact, a CW beam was chosen so that there would be no timing considerations between the pump and the signal beam. The signal beam has now been transformed into a pulsed format by the BEFWM process and is timed appropriately for SBS amplification. Several stages of SBS amplification can now be used to generate enough energy in this signal conjugate beam for adequate detection. The now pulsed conjugate signal beam is transmitted back to the target once again. Again, it is diffusely reflected from the target area, however this time a much larger fraction of the light will return to the original transmitting telescope than would be expected from linear light propagation. This is due to the phase conjugate properties of the light. The light can now be detected with little or no speckle noise. 


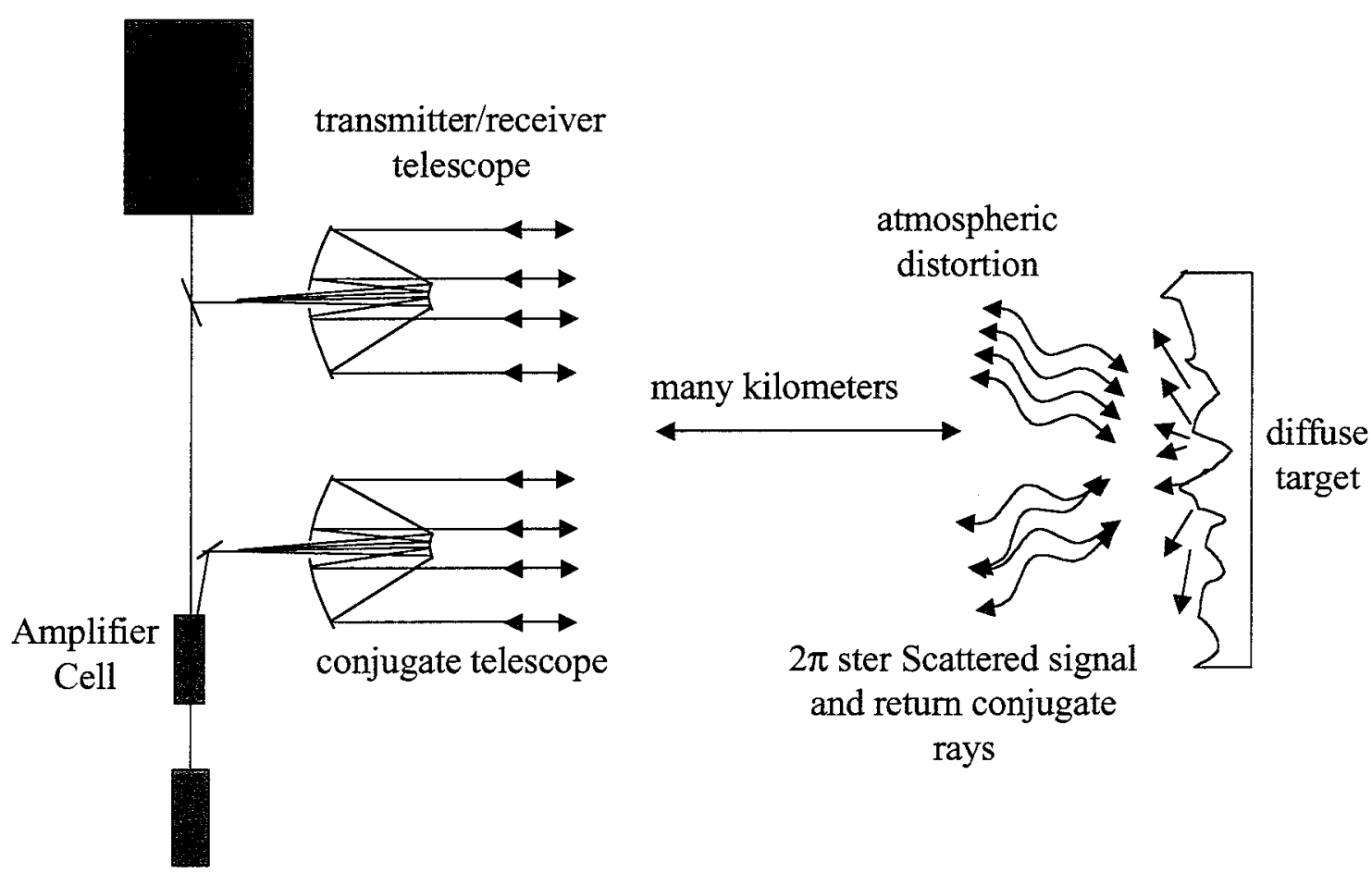

Figure 2. The two-round-trip optical phase conjugation system. See text for a detailed description.

\section{EXPERIMENTS}

Numerical simulations of the nonlinear optical processes were first performed in order to gain a better understanding of the envelope of operation of the optical phase conjugation process as applied towards DIAL systems. The initial modeling was performed by Ted Scharlemann of LLNL s NAI directorate using the same code that is normally used to model other LIDAR systems, Figure 3 . The $10 \mathrm{~km}$ distance to the topographic background and the 1 meter aperture detector resulted in a loss of $70 \mathrm{~dB}$ in the return signal information and power, not normally a regime in which optical phase conjugation is considered practical. From this numerical study it was determined that the quality of the phase conjugation process is directly dependent upon the number of speckle bright spots, called speckle cells, in the detection aperture.

A laboratory experiment was planned that would closely approximate an outdoor field LIDAR system. A numerical study was performed using a similar code to the one described above to assure that the detection aperture would have adequate phase conjugation quality. The numerical study determined that the number of speckle cells in the collection aperture determines the phase conjugate quality. Usually 20 or more speckle cells is adequate for good conjugate fidelity. The number of speckle cells is a controllable parameter, determined only by the characteristics of the LIDAR itself, not on the properties of the target used. The experiment was planned using a Nd:YAG laser 
(a)

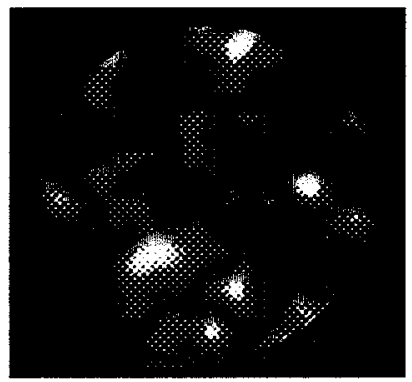

(b)

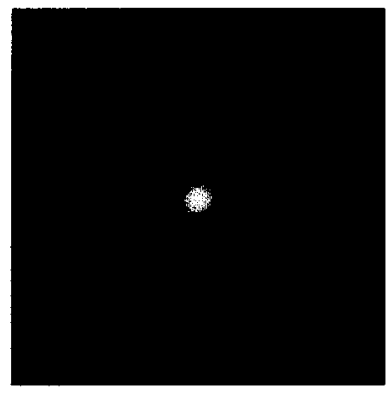

Figure 3. Modelled speckle pattern (a) and phase conjugate return (b) of a LIDAR signal from a 1 meter aperture LIDAR and $10 \mathrm{~km}$ to the topographic target.

system and a carbon disulfide filled cell as the nonlinear SBS material. The results of this initial experiment, figure $4 \mathrm{a}$ and $\mathrm{b}$, showed that the SBS process significantly improved the quality of the scattered light. The single pulse $\mathrm{S} / \mathrm{N}$ improvement for this system was 100:1 and the multiple pulse $\mathrm{S} / \mathrm{N}$ improvement with averaging was 9.3:1. The SBS gain was $10 \mathrm{~dB}$, lower than estimated above due to the bulk focusing optics used, but adequate for the laboratory testing used here. Although good, the results were less than expected through the numerical studies performed earlier. The problem was found to be in the geometry of the SBS phase conjugator. Although the geometry is the one most commonly used for SBS phase conjugation, it was not appropriate for fields with a large number of speckle cells. The geometry prevented light rays that were entering at extreme angles from participating in the phase conjugation process. Thus, some of the information was lost and incomplete phase conjugation resulted. We were able to reproduce this effect numerically as shown in figure $4 \mathrm{c}$ and $\mathrm{d}$.

The speckle noise reduction was measured in by measuring the returned energy

(a)

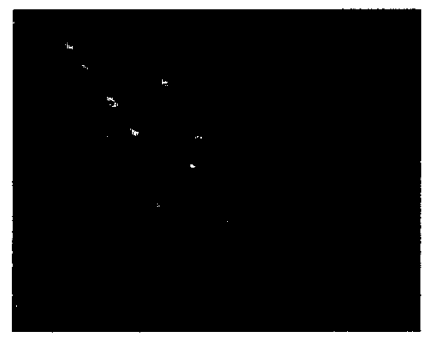

(c)

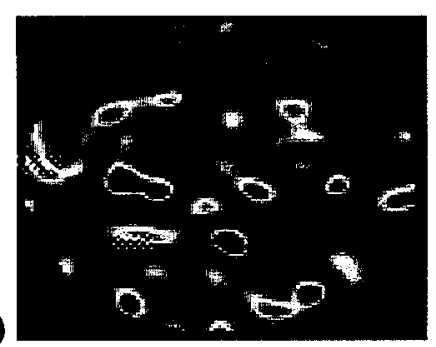

(b)

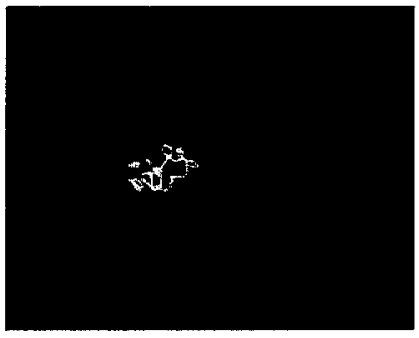

(d)

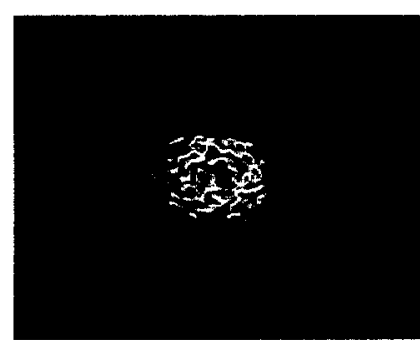

Figure 4. The speckle return (a) in the laboratory experiment. The return (b) has much less speckle noise when the phase conjugation system is inserted. The numerical simulations (c) and (d) accurately predict the behavior seen in (a) and (b) respectively. 
contained within an aperture that was placed in the return beam path at the focus of the receiving telescope. The energy was measured for multiple laser pulses while the characteristics of the target were changed between each pulse to simulate the constantly changing conditions of the atmosphere, figure 5 . The signal-to-noise $(\mathrm{S} / \mathrm{N})$ improvement when using the SBS phase conjugation system was 9.3. This represents almost an order of magnitude improvement in the ability of a LIDAR system to detect a chemical or two orders of magnitude decrease in averaging time that results in the same $\mathrm{S} / \mathrm{N}$ as would be without the phase conjugation system.

(a)

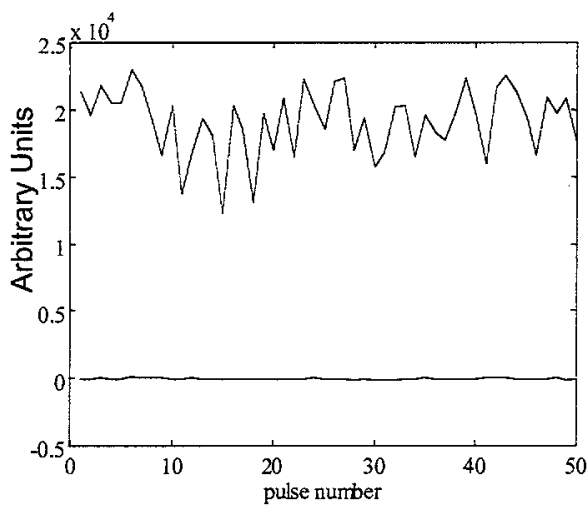

(b)

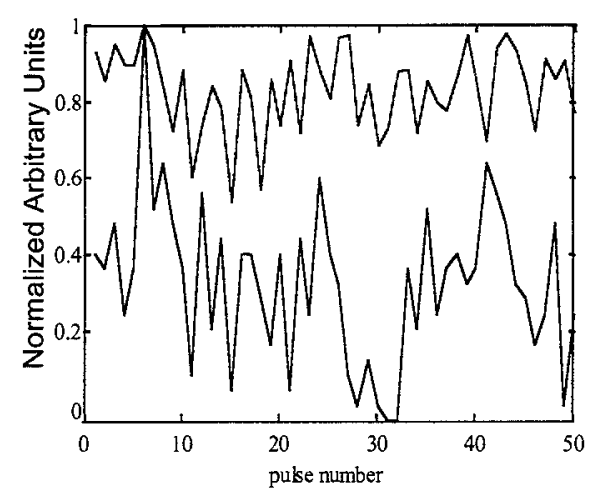

Figure 5. The raw (a) and normalized (b) signal return without (red) and with (blue) optical phase conjugation.

Waveguides, which can capture the light rays that enter at extreme angles, were one solution to the problem of incomplete phase conjugation that was discussed previously. The waveguides are cylindrical pipes or fibers that are filled with an SBS active material. Liquid and solid filled waveguides were then tested for their applicability to this problem. The liquid filled waveguides did have good phase conjugation properties. These waveguides, however, suffered from heat buildup near the entrance that created bubbles after several minutes of operation. The bubbles would be drawn into the waveguide, stopping the SBS process. This problem can be corrected by flowing the liquid continuously through the waveguide, removing bubbles whenever they form.

The solid waveguides are essentially optical fibers that are specially shaped to enhance the SBS process. These waveguides have similar phase conjugation properties to those of the liquid filled counterparts, but do not suffer from the bubble formation. For the incorporation of the waveguide SBS generators into a LIDAR system it is essential that they reach SBS threshold well below the power levels available. Since the power available is approximately $600 \mu \mathrm{J}$ for the oscillator/generator the threshold should be below $100 \mu \mathrm{J}$ to achieve adequate conjugate intensity. Low thresholds for SBS initiation, as low as $70 \mu \mathrm{J}$, were demonstrated as part of this project thus far. This is an enhancement over the original bulk carbon-disulfide cells that had a threshold of more than $2 \mathrm{~mJ}$. The maximum power permissible in the fibers tested is limited to twice the threshold value, too low to yield sufficient energy for the speckle correction testing. New 
fibers that have an improved geometry for SBS enhancement were ordered and should arrive soon. The threshold for these new fibers has been calculated to be better then 10 $\mu \mathrm{J}$ with a maximum of power of several millijoules.

A collaborative effort, funded through this project, with the nonlinear optics group at the University of Rochester, to search for appropriate materials for the SBS process in wavelength regions of interest to most commercial DIAL systems was performed. The most desirable wavelength region is that from 2 to $4 \mu \mathrm{m}$. A table of more than 100 suitable materials was found and narrowed down to the best candidates. From this list it was evident that commercially available infrared (IR) fibers would be suitable candidates due to their availability in a waveguide form. IR sapphire fibers can be commercially manufactured to our desired geometry while maintaining a high damage threshold. The calculated theoretical threshold for SBS initiation in these fibers was well within the capabilities of the laser systems in the NAI LIDAR facilities. We acquired several tapered sapphire fibers for testing. We began the tests at $1064 \mathrm{~nm}$ since SBS at that wavelength had previously been achieved in this project yet phase conjugation at the longer wavelengths $(>1.5 \mu \mathrm{m})$ used in the DIAL LIDAR facilities have not been demonstrated readily even in the literature in fiber geometries. However, SBS was not seen in the sapphire fibers even at pulse energies more than an order of magnitude higher than was calculated. It is assumed that the single crystal structure of the sapphire fibers suppresses SBS. We are now collaborating with Professor Boyd at the University of Rochester to research this phenomenon for possible applications to SBS suppression in telecommunications and high power transmission through fibers.

Silica fibers have been studied in the literature for applications in SBS optical phase conjugation. This early fiber work relied heavily upon the fibers ability to reproduce desirable SBS characteristics, such as long interaction regions [2] and an oscillator/amplifier configuration through a tapered geometry [3]. It was decided that we would use silica fibers for the next phase of work even though they do not have adequate transmission in the IR for DIAL LIDAR work at wavelengths greater than $2.5 \mu \mathrm{m}$ and had marginal angular acceptance for this project. Recent work $[4,5]$ using tapered optical fibers show mixed success. Heuer et. al. showed $>90 \%$ reflectivity and good fidelity, while Eichler, et. al. had $<65 \%$ reflectivity. The geometries and experimental conditions were similar. The low reflectivity in ref. 2 is described to be due to the amplitude fluctuations of the return beam. However, these amplitude fluctuations are well understood [6] to be due to the reflections off the fiber ends. In that case, the reflectivity of ref. 1 should also have suffered low reflectivity. In this paper, we study a long taper and a short taper to find the reason for this discrepancy.

We chose two fibers, a $6 \mathrm{~m}$ long silica fiber with a $3 \mathrm{~m}$ taper from $300 \mu \mathrm{m}$ to 100 $\mu \mathrm{m}$ taper over the first 3 meters of the fiber [7]; and a 2 meter long silica fiber with a constant $50 \mu \mathrm{m}$ diameter core for the first meter leading to a $5 \mathrm{~cm}$ long taper down to a $15 \mu \mathrm{m}$ core region for a length of $1 \mathrm{~m}$. The material numerical aperture (NA) of the fibers was 0.27 and 0.37 respectively. The second fiber was a specialty fiber made at the University of Bath in collaboration with this project. Conventionally, fibers are "tapered" - locally reduced in diameter - by heating and stretching in a small heat source. The fiber is typically pulled symmetrically. Variant techniques allow the heat source to oscillate or pull only one end of the fiber, but the process is fundamentally the same: both ends of the fiber are drawn away from a central hot zone, and the fiber diameter decreases with time. 
Such tapered fibers can have very low loss, and are the basis for optical devices such as fiber beamsplitters. The length of fiber that can be narrowed is however limited to a few centimeters.

In contrast, the tapered fiber in our experiments was made continuously by a new technique, using effectively a miniature fiber drawing tower. The precursor fiber (like the preform in a fiber drawing tower) is fed into the hot zone, and a fiber of reduced diameter is drawn out at a faster rate. The final steady-state diameter is determined only by the ratio of the feed and draw rates, and is constant with time. The length of narrowed fiber produced is in principle unlimited.

We implemented this "mini drawing tower" using two rotating wheels (superficially resembling a reel-to-reel tape recorder) driven by stepper motors to feed and draw the fiber. The precursor fiber was stripped of its coating over sufficient length and wrapped around the feed spool. One end was attached to the draw spool. An oxybutane flame was introduced at the mid-point, and the spools rotated to give a feed rate of $0.1 \mathrm{~mm} \mathrm{~s}^{-1}$ and a draw rate of $1.1 \mathrm{~mm} \mathrm{~s}^{-1}$. This reduced the diameter of the fiber's core from $50 \mathrm{~m}$ to $15 \mathrm{~m}$. After $1 \mathrm{~m}$ of $15 \mathrm{~m}$ fiber was drawn, the feed rate was steadily increased to match the draw rate, forming a gradual taper transition about $50 \mathrm{~mm}$ long between the $15 \mathrm{~m}$ and $50 \mathrm{~m}$ fibers. The flame was then removed and the motors stopped.

We first used low power $1064 \mathrm{~nm}$ and then $1530 \mathrm{~nm}$ to measure the effective numerical aperture of the fibers. The $1064 \mathrm{~nm}$ light was generated using a Diode pumped Nd:YAG oscillator. The pulse was gaussian in both time and space with a full-width at half-maximum (FHWM) duration of $30 \mathrm{~ns}$ and a $1 / \mathrm{e}^{2}$ diameter of $3 \mathrm{~mm}$. The beam was nearly diffraction limited with a $\mathrm{M}^{2}<1.2$. The $1530 \mathrm{~nm}$ pulse was generated using a periodically-poled Lithium Niobate crystal OPO. The saturation in the OPO lead to a longer duration than the pump with a FWHM of $35 \mathrm{~ns}$. The pulse quality was degraded by the OPO leading to an $\mathrm{M}^{2}<2$ for all pulses. The $1530 \mathrm{~nm}$ pulse shape would change occasionally due to thermal changes in the OPO. The phonon decay time, $10 \mathrm{~ns}$ and 21 $\mathrm{ns}$ for $1063 \mathrm{~nm}$ and $1530 \mathrm{~nm}$ respectively, was less than the pulse duration for both wavelengths, however, the rising edge of the $1530 \mathrm{~nm}$ pulse was substantially faster than the phonon lifetime. The beams were focused down through a focus and allowed to expand to $80 \%$ the diameter of the core of the fiber being tested using a $100 \mathrm{~mm}$ focal length lens at $1064 \mathrm{~nm}$ and a $63 \mathrm{~mm}$ focal length lens at $1530 \mathrm{~nm}$. The angular divergence of this input pump beams was then $15 \mathrm{mrad}$ and $25 \mathrm{mrad}$ respectively. The fiber was first placed normal to this input beam and then scanned in angle to map out the transmission as a function of input angle. Care was taken to assure that the input beam remained centered on the fiber core as the angle was scanned. The output spatial pattern in each case was an annular ring or a disk demonstrating that this excited modes that propagated only within certain angular limits. The results for both fibers as a function of angle can be seen in figure 6 . As was expected, the transmission through each fiber went to zero as the angle exceeded the material NA, divided by the ratio of the core sizes before and after the taper. Unlike normal step-index fibers the cutoff angle is gradual.

The second test was similar to the first with the exception of increasing the incident power at each angle to find the SBS threshold. The threshold condition at 1530 $\mathrm{nm}$ was difficult to determine compared to the $1064 \mathrm{~nm}$ results. This is due to the larger speckle return from inside the fiber and to the fast leading edge of the pulse from the 
OPO as discussed above. The results for each fiber are shown in figure 7. The shape of the curves is similar to that shown in figure 6. This shows that higher order modes experience more attenuation in the fiber before the oscillator section and thus will not phase conjugate efficiently, even though they are well within the calculated effective NA of the fiber. Even though the higher order modes may not have any reflectance from the oscillator section of the fiber, they will experience a Brillouin-enhanced four-wavemixing interaction with the lower order modes, when present, in the amplifier section and thus still show some reflectivity in those cases. This reflectivity, however, is reduced from that demonstrated by the lowest order modes. All modes will experience some loss in the taper section and will therefore be affected. Therefore, only moderately aberrated beams can be conjugated with high fidelity. At this point that it was theorized that beams with large divergence angles will have a higher threshold and lower saturation fluence than beams with low divergence. The higher order modes experience attenuation along the taper and into the oscillator section. This attenuation is similar in both the long and short tapers. Thus, the shorter taper will allow the higher order modes to extend into regions of smaller core diameters, increasing the reflectivity somewhat. It is expected, therefore, that the shorter taped fiber will demonstrate higher reflectivity than the longer taper.

Finally, the power was increased to more than 10 times threshold at $1064 \mathrm{~nm}$ in each fiber with first a small input beam divergence and then a large input divergence. We were not able to perform this test at $1530 \mathrm{~nm}$ because of the OPO output limit of 1 $\mathrm{mJ}$. The results of this test are shown in figure 8. The solid lines drawn are a best fit to a simple SBS theory with the addition of a loss term [8]. The maximum reflectivity of both the long and the short taper were similar when the input beam divergence was small compared to the effective NA of each fiber. When the input beam divergence was comparable to effective NA of the fiber, the maximum reflectivity of the long taper was less than that of the short taper. The discrepancy in the curve fit is due to the four-wave mixing processes that are occurring when some of the input energy does not reach the oscillator region of the fiber. It is apparent that the relatively flat transmission profile of the $300 \mu \mathrm{m}$ fiber taper results in a simple SBS conjugation process. The more complex shape of the $50 \mu \mathrm{m}$ taper transmission profile with respect to angle does not allow for a simple SBS process to occur, and thus will always have a four-wave mixing process occurring. This can still occur efficiently in the short taper since a substantial fraction of the attenuated energy will still enter the high field region of the oscillator, where the fourwave mixing process can occur more efficiently. 


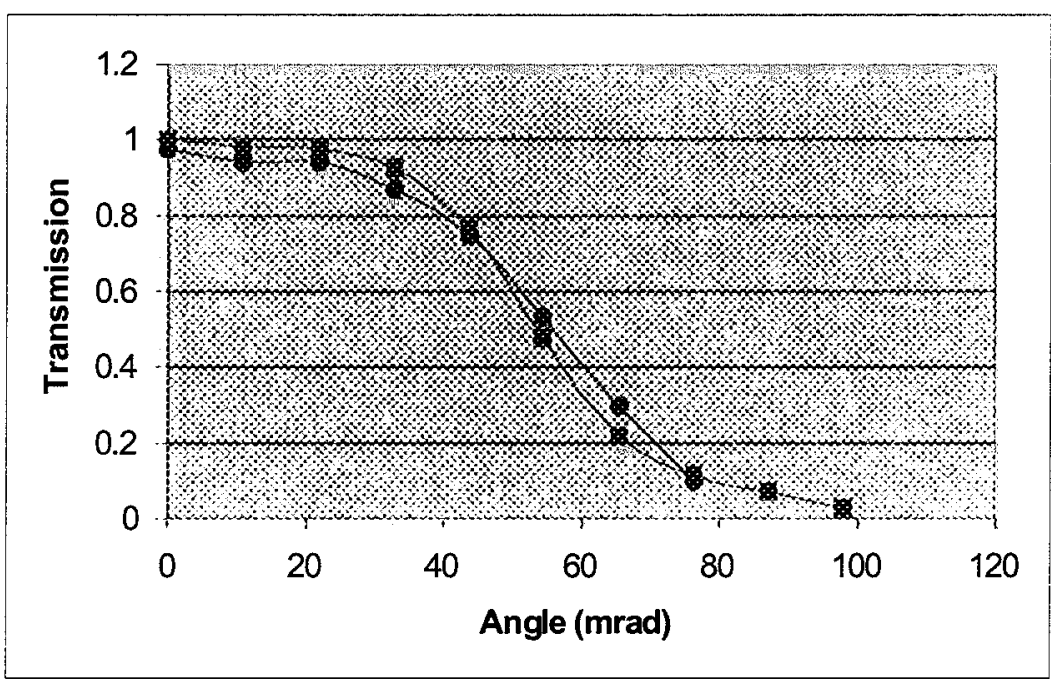

(a)

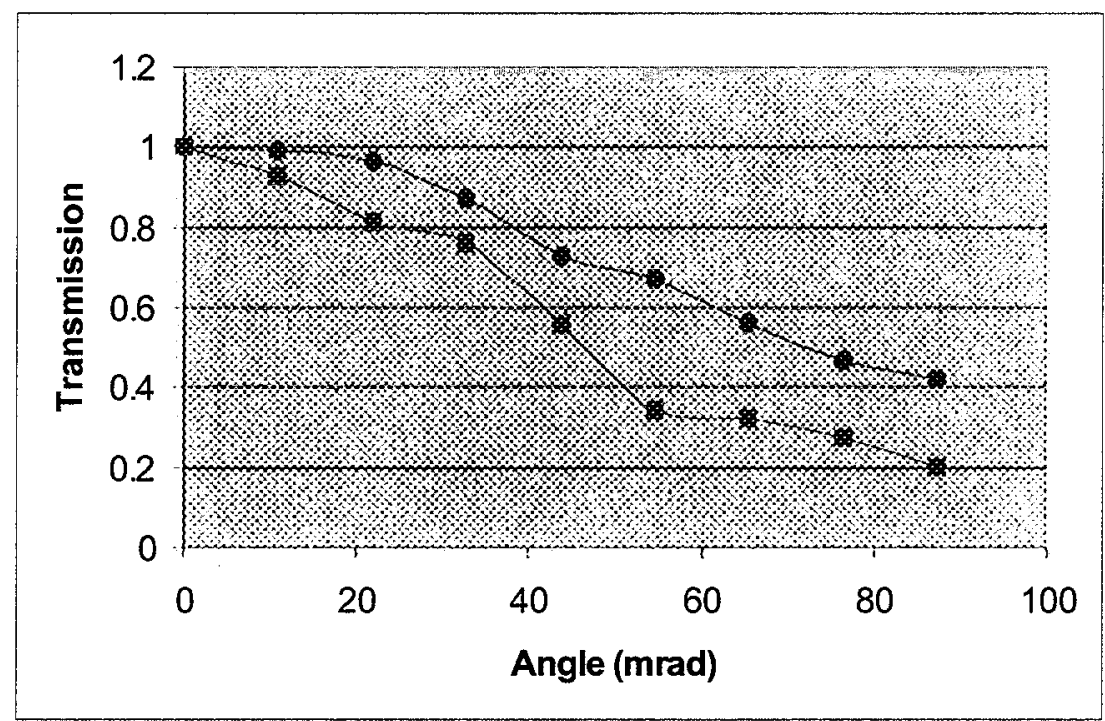

(b)

Figure 6. The transmission of the (a) 300 to $100 \mathrm{~mm}$ taper and (b) the $50 \mathrm{~mm}$ to $15 \mathrm{~mm}$ taper. The Fresnel reflections are not included in this data. Circles are at $1064 \mathrm{~nm}$ and squares are at $1530 \mathrm{~nm}$. The lines drawn are to serve as aids to the eye. 


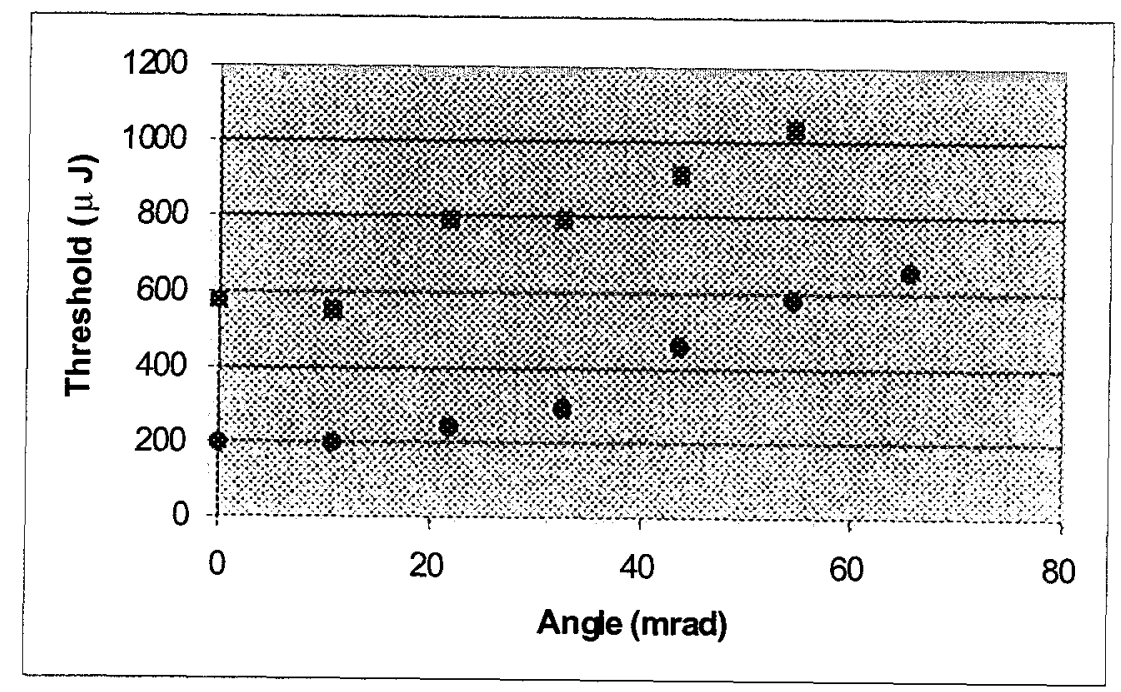

(a)

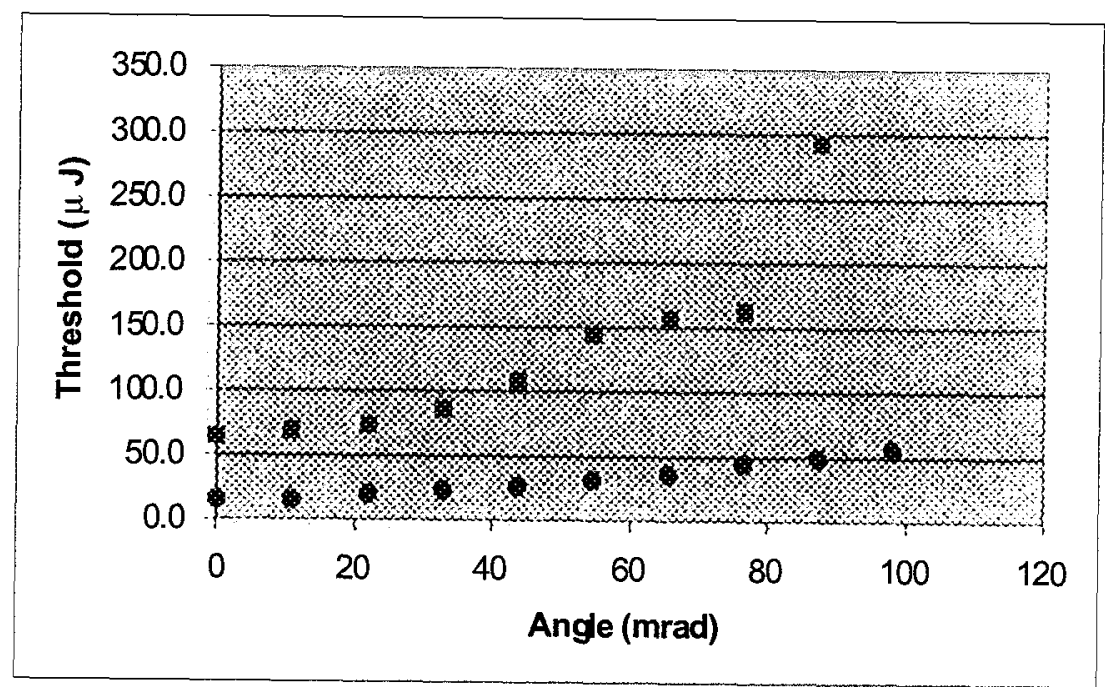

(b)

Figure 7. SBS threshold for (a) the $300 \mu \mathrm{m}$ to $100 \mu \mathrm{m}$ taper and (b) for the $50 \mu \mathrm{m}$ to the $15 \mu \mathrm{m}$ taper. Squares are for $1064 \mathrm{~nm}$ and circles are for $1530 \mathrm{~nm}$. 


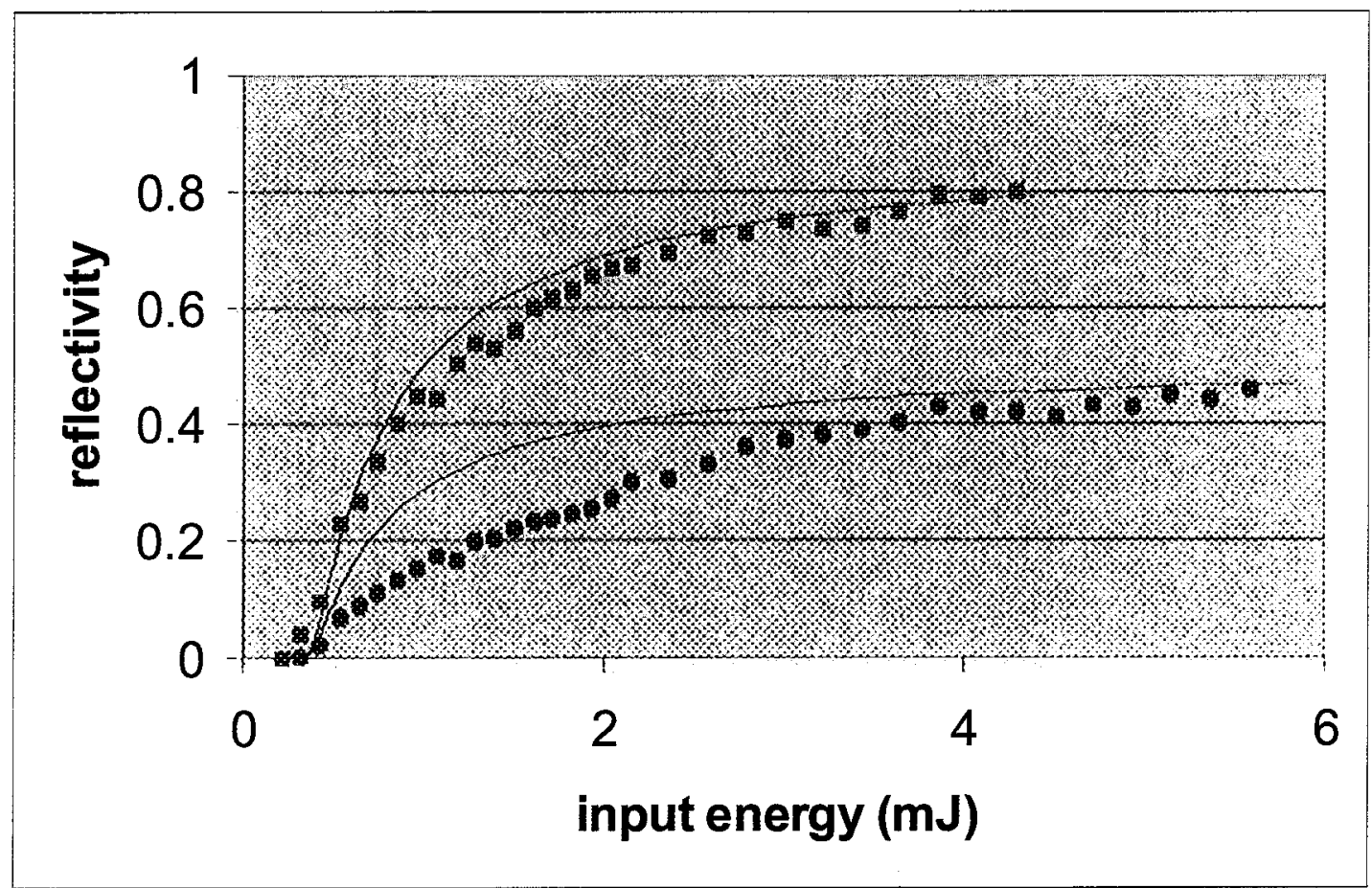

(a)

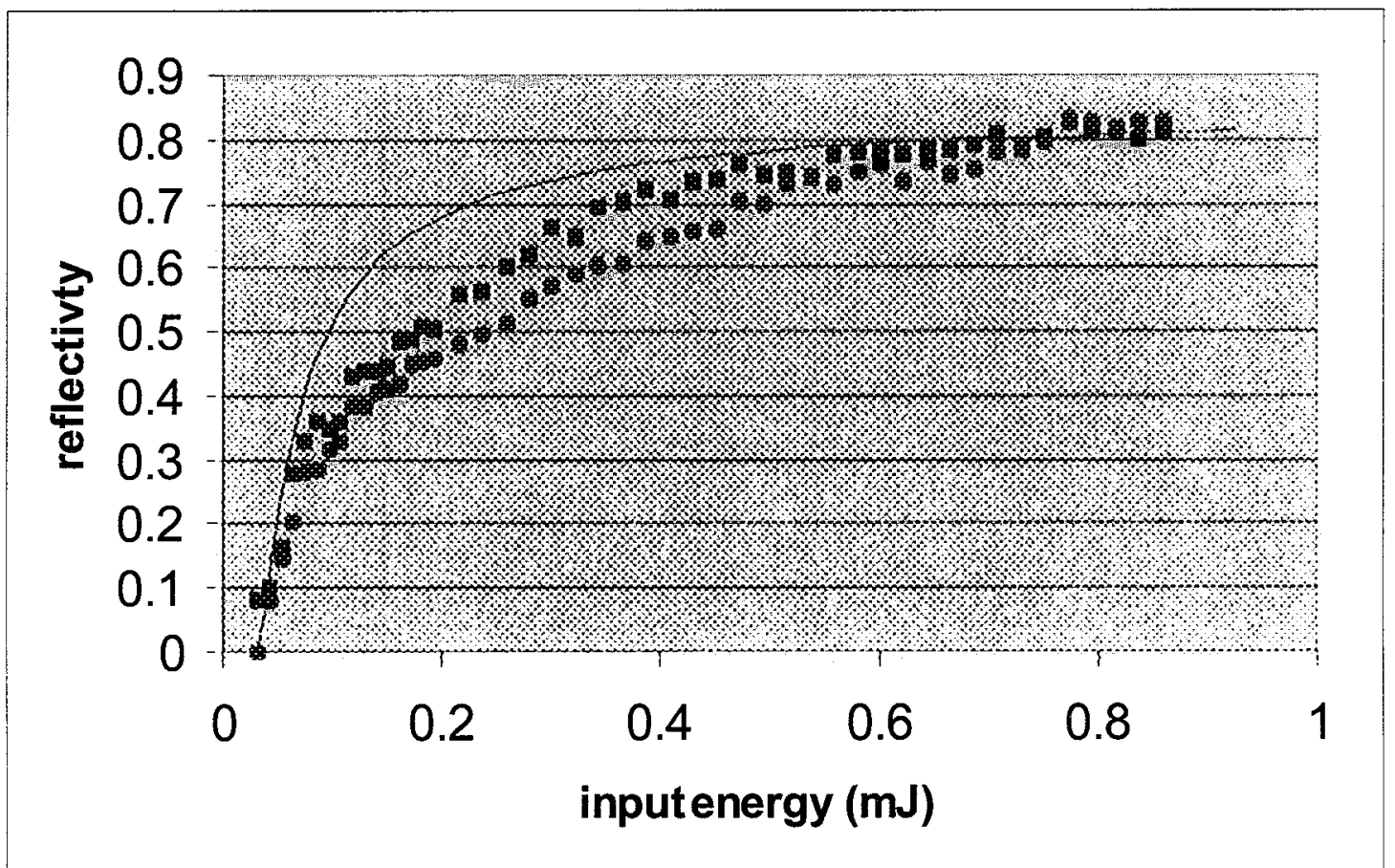

(b)

Figure 8. The reflectivity as a function of pump energy for the (a) 300 to $100 \mathrm{~mm}$ taper, and (b) the $50 \mathrm{~mm}$ to $15 \mathrm{~mm}$ taper. The circles are for the low na input and the squares are the high na input. 
This test showed that these fused silica tapered fibers still displayed similar characteristics to the bulk SBS system that we were originally using. Non-tapered fibers have a discrete cutoff. All angles lower that the cutoff propagate, those above leak. The tapered fibers show attenuation of almost all modes. The ones nearest to the angular cutoff had more attenuation than those further away. This leads to angular frequency filtering, destroying the phase conjugate abilities of the fiber for large divergence beams, such as ours. Non-tapered fibers would also not work due to the large aperture required to capture the full beam. This large aperture would require prohibitively large energies in the SBS pump beam in order to reach threshold. Thus, these fibers were not able to be used in the system as well.

On the other hand, fibers that have large input NA s $(\sim 1)$, such as the sapphire fibers, would easily meet all of the requirements for angular acceptance and reduction ratios. We now need to proceed with another study to investigate other potential fiber materials that a polycrystalline so that they support the SBS process, transmit well in the mid-IR, and can be easily tapered for the optimal geometry for tapered fiber SBS.

\section{CONCLUSIONS}

During the course of this project we were able to show that optical phase conjugation can, under certain conditions, improve the S/N of DIAL LIDAR signals. We were able to demonstrate this in the lab as well in computer simulations. We were able to phase conjugate $1530 \mathrm{~nm}$ light in optical fibers, for what we believe to be the first time anyone has done this with nanosecond scale pulses. We also demonstrated the large numerical aperture fibers with appropriate tapers necessary for the optical phase conjugation system to be compact and yet still acquire the DIAL signal. However, due to the problems with the sapphire fiber crystalline structure and the low numerical aperture of the silica fibers, we were not able to demonstrate this system at the $1 \mathrm{~km}$ distances that we had anticipated. We do believe, however, that we have paved the way for this technology to be successful in the near future. We are working with NN-20 and others for follow-on funding to expand the scope of the work that was done on this project and to find appropriate fibers that will facilitate the transfer of this technology to DIAL LIDAR systems in the field.

\section{References:}

1. See for example Fisher, R. A. ed. Optical Phase Conjugation, Academic Press, Orlando Fl. (1983) sections chap. 6-7

2. See for example Hon, D. T., Pulse compression by stimulated Brillouin scattering, Opt. Lett., 5, 516-518, (1980)

3. See for example Vasil ev, A. F., Yashin, V. E., Stimulated Brillouin scattering at high values of the excess of the pump energy above the threshold, Sov. J. Qaun. Elec., 17, 644-647 (1987)

4. Heuer, A. Menzel, R. Phase-conjugating stimulated Brillouin scattering mirror for low powers and reflectivites above $90 \%$ in an internally tapered optical fiber, Opt. Lett, 23, 834-836 (1998) 
5. Eichler, J. Liu, B., Wittler, O. Fiber phase conjugators: time- and frequencyresolved reflectivity, Conf. On Lasers and Elec., 144 (1998)

6. Agrawal, G. P., Noninear Fiber Optics, Berkely academic press, (1989)

7. Fiber produced by Fiberguide industries as a standard part.

8. Boyd, R. W., Nonlinear Optics, San Diego Academic Press (1992) 


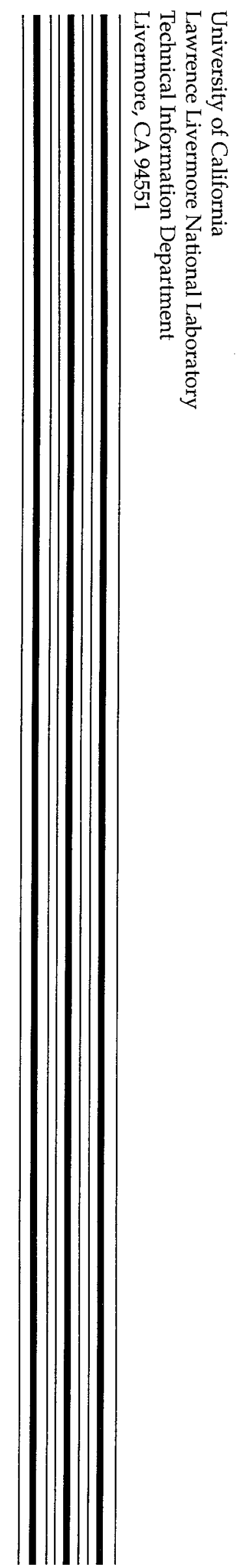

